\title{
NOTES
}

\section{Influence of Stannic Chloride on the Copolymerization Behaviour of Vinyl Acetate with Acrylonitrile}

\author{
S. M. Mokhtar, S. M. Abdel-Aziz, and Abo El-Khair B. Mostafa* \\ Chemistry Department, University College for Women, \\ Ain Shams University, Cairo, A.R.E.
}

(Received April 26, 1988)

\begin{abstract}
KEY WORDS Copolymerization / Vinyl Acetate-Acrylonitrile / Stannic Chloride / Copolymerization Rate / Reactivity Ratios / Copolymer Structure
\end{abstract}

Free radical polymerization is one of the most important methods of obtaining synthetic polymers. However, the polymerization reaction by free radical mechanism cannot be controlled easily, and this is the main disadvantage of this process. As is known, the propagation reaction affects the rate of the overall polymerization process, the molecular weight of the polymer, the configuration of the macromolecules and the composition of the obtained copolymer. Extensive studies have been published on homo- and copolymerization of vinyl monomers in presence of some inorganic salts, such as: $\mathrm{LiCl}, \mathrm{ZnCl}_{2}, \mathrm{AlBr}_{3}$ and $\mathrm{SnCl}_{4} \cdot{ }^{1-3}$ These salts form complexes with functional groups of such monomers and show considerable effect on the reaction rate and composition of the resulting polymers. ${ }^{4}$

In the present work the effect of $\mathrm{SnCl}_{4}$ as a modifier on the copolymerization behaviour of vinyl acetate (VAc) and acrylonitrile (AN) in bulk was investigated. The study was performed by measuring the copolymerization rates, the structure of the obtained copolymers and by determining the copolymerization parameters.

Copolymerization rates were measured using a dilatometer. The copolymer compositions were calculated on the basis of nitrogen content of the copolymers. Copolymerization parameters were calculated by the FinemanRoss method ${ }^{5}$ and the Kelen-Tüdos method. ${ }^{6}$ The infrared spectra were recorded by a double beam spectrophotometer Perkin-Elmer 398. X-ray diffraction patterns were obtained by philips apparatus (pW 1390 channel control and pW 1373 goniometer supply) using a $\mathrm{Cu}$ $K_{\alpha}$ beam by the film method.

Figure 1 illustrates the results of the percent conversion-time for the copolymerizations of vinyl acetate (VAc) and acrylonitrile (AN) of 20 to $80 \mathrm{~mol} \%$ at various concentrations of $\mathrm{SnCl}_{4}$. It was found that as the concentration of $\mathrm{SnCl}_{4}$ increased, the overall rate of the reaction increased. Figure 2 shows the relative rates of VAc and $\mathrm{AN}$ copolymerization in the absence and presence of $\mathrm{SnCl}_{4}$. Obviously, the relative rate of the copolymerization reaction increases about 19 times with increasing $\mathrm{SnCl}_{4}$ content in the reaction medium from 0.0 to 0.249 in molar ratio. These results indicate that $\mathrm{SnCl}_{4}$ is more effective on the reaction kinetics of VAc-AN copolymerization than $\mathrm{ZnCl}_{2}{ }^{4}$ Similar behaviour was also observed for the

* To whom all correspondence should be addressed. 


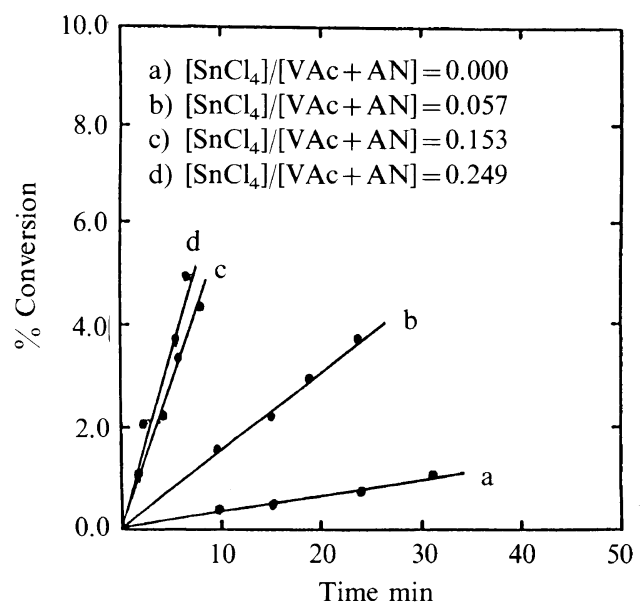

Figure 1. Percent conversion-time curves for bulk copolymerization of vinyl acetate with acrylonitrile (20 to $80 \mathrm{~mol} \%$ ) initiated by azobisisobutyronitrile in the presence of $\mathrm{SnCl}_{4}$ at $65^{\circ} \mathrm{C}$.

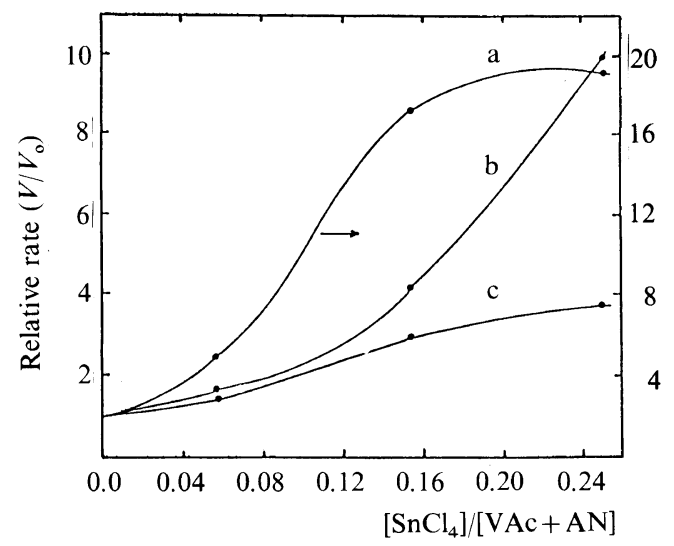

Figure 2. Dependence of the relative rates of VAc-AN copolymerization in the presence of azobisisobutyronitrile initiator on $\mathrm{SnCl}_{4}$ concentration at $65^{\circ} \mathrm{C}$ : a, [VAc] $[\mathrm{AN}]=20$ to $80 \mathrm{~mol} \%$; b, [VAc] $/[\mathrm{AN}]=50$ to $50 \mathrm{~mol} \%$; , [VAc] $/[\mathrm{AN}]=70$ to $30 \mathrm{~mol} \%$.

copolymerization system of VAc and AN of 50 to 50 and of 70 to $30 \mathrm{~mol}^{\%}$, respectively ( $c f$. Figures 2 and 3). In addition the copolymerization reaction of $\mathrm{VAc}$ and $\mathrm{AN}$ is more efficient to produce a copolymer with a higher rate with increasing the content of both $\mathrm{AN}$ and $\mathrm{SnCl}_{4}$ in the monomer feed. The same trend was reported previously for the copolymerizations of some vinyl monomers in

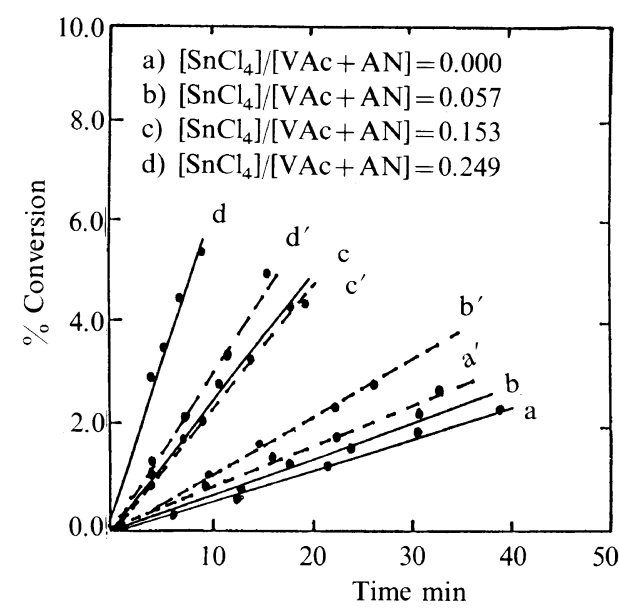

Figure 3. Percent conversion-time curves for bulk copolymerization of vinyl acetate with acrylonitrile initiated by azobisisobutyronitrile in the presence of $\mathrm{SnCl}_{4}$ at $65^{\circ} \mathrm{C}:-,[\mathrm{VAc}] /[\mathrm{AN}]=50$ to $50 \mathrm{~mol} \%$; --- , $[\mathrm{VAc}] /[\mathrm{AN}]=70$ to $30 \mathrm{~mol} \%$.

presence of Lewis acids. ${ }^{3}$

The effect of $\mathrm{SnCl}_{4}$ on the reactivity behaviour of comonomers during their copolymerization can be confirmed from IR spectra. The IR spectrum of VAc in presence of $\mathrm{SnCl}_{4}$ shows that there is a shift in the frequency of the absorption band of the ester group from $1760 \mathrm{~cm}^{-1}$ to $1755 \mathrm{~cm}^{-1}$. Also, the absorption band of nitrile group of $\mathrm{AN}$ at $2270 \mathrm{~cm}^{-1}$ undergoes a shift to $2260 \mathrm{~cm}^{-1}$ in presence of $\mathrm{SnCl}_{4}$. These results suggest that $\mathrm{SnCl}_{4}$ forms complex with the functional group of each monomer. Thus, it raises their polarity, leading to increased conjugation energy of the $\pi$-electron of the $\mathrm{C}=\mathrm{C}$ of the vinyl group. Consequently, $\mathrm{SnCl}_{4}$ increases the reactivity of the two monomers, towards the copolymerization reaction.

The action of $\mathrm{SnCl}_{4}$ on the structure of the resulting VAc-AN copolymer was studied by $\mathrm{X}$-ray measurements. Figure 4 shows the Xray diffraction diagrams for the copolymer samples obtained during the copolymerization of VAc with AN of 30 to 70 with various concentrations of $\mathrm{SnCl}_{4}$. It was observed that in the absence of $\mathrm{SnCl}_{4}$ strong reflections 


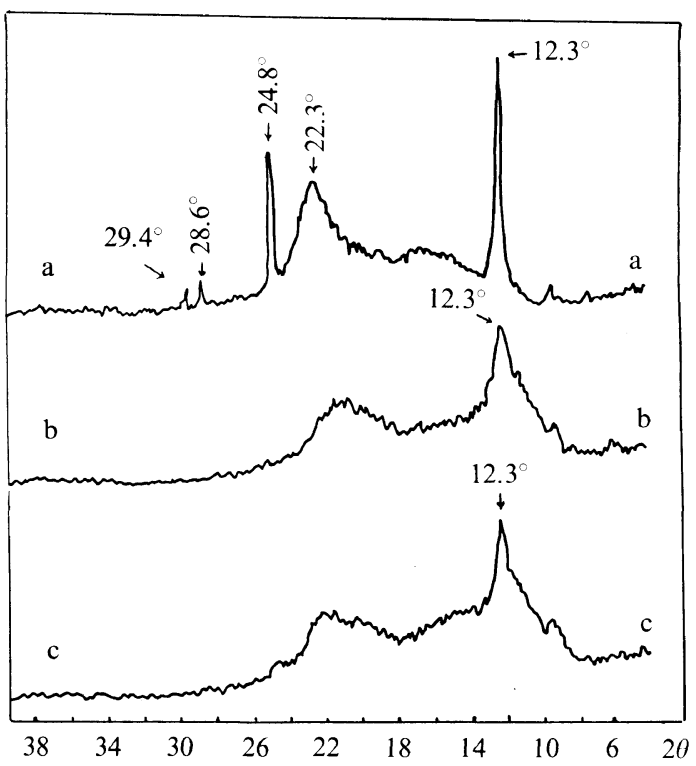

Figure 4. X-Ray diffraction curves of vinyl acetate and acrylonitrile copolymer formed during copolymerization of VAc with AN of 30 to 70 in the presence of $\mathrm{SnCl}_{4}$ at $65^{\circ} \mathrm{C}$ using azobisisobutyronitrile as an initiator: a, $\left[\mathrm{SnCl}_{4}\right] /[\mathrm{VAc}+\mathrm{AN}]=0.00 ; \quad \mathrm{b}, \quad\left[\mathrm{SnCl}_{4}\right] /[\mathrm{VAc}+\mathrm{AN}]=$ $0.153 ; \mathrm{c},\left[\mathrm{SnCl}_{4}\right] /[\mathrm{VAc}+\mathrm{AN}]=0.249$.

appeared at $2 \theta=12.3^{\circ}, 22.3$ and $24.8^{\circ}$ and small reflections appeared at $2 \theta=28.6^{\circ}$ and $29.4^{\circ}$ (Figure 4a). However in presence of $\mathrm{SnCl}_{4}$ (Figures $4 \mathrm{~b}$ and $4 \mathrm{c}$ ), the reflection at $2 \theta=22.3^{\circ}$ and above this range disappeared and a new broaden area appeared with a maximum reflection at about $2 \theta=21^{\circ}$. Therefore, $\mathrm{SnCl}_{4}$ may affect the structure of the obtained VAc-AN copolymer, i.e., order of monomeric unit arrangements in the copolymer chains. Consequently, it affects the properties of the resultant copolymers. This is very important and useful in industry, since VAc-AN copolymers are used for the manufacture of membranes ${ }^{7}$ and preparing fibrous activated carbon of high adsorption capacity and strength. ${ }^{8}$

The compositions of the comonomers and resultant copolymers at the initial stages of polymerization, i.e., $<10 \%$ conversion, for the copolymerizing system of $\mathrm{VAc}$ and $\mathrm{AN}$ in

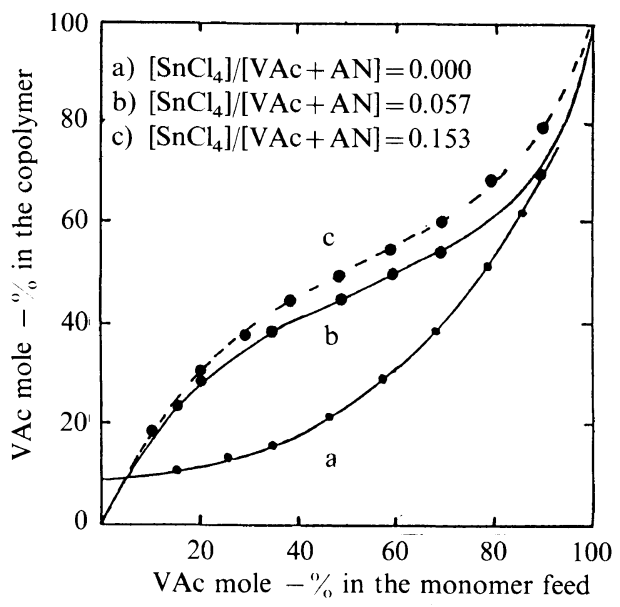

Figure 5. Monomer-copolymer composition curves for vinyl acetate with acrylonitrile copolymerization in the presence of $\mathrm{SnCl}_{4}$ initiated by azobisisobutyronitrile $\left([\mathrm{AIBN}]=5 \times 10^{-3} \mathrm{moll}^{-1}\right)$ at $65^{\circ} \mathrm{C}$.

Table I. Apparent reactivity ratios for the bulk copolymerization of vinyl acetate (VAc) and acrylonitrile $(\mathrm{AN})$ in different concentrations of $\mathrm{SnCl}_{4}$ at $65^{\circ} \mathrm{C}$

\begin{tabular}{ccccccc}
\hline \multirow{2}{*}[\mathrm{SnCl}_{4}]{} & & \multicolumn{2}{c}{ Fineman-Ross } & & \multicolumn{2}{c}{ Kelen-Tüdos } \\
\cline { 1 - 1 } \cline { 6 - 7 }$[\mathrm{VAc}+\mathrm{AN}]$ & & $r_{\mathrm{VAc}}$ & $r_{\mathrm{AN}}$ & & $r_{\mathrm{VAc}}$ & $r_{\mathrm{AN}}$ \\
\hline 0.00 & 0.044 & 2.110 & & 0.045 & 2.340 \\
0.057 & 0.180 & 0.358 & & 0.180 & 0.359 \\
0.153 & 0.373 & 0.370 & & 0.370 & 0.370 \\
\hline
\end{tabular}

${ }^{a}$ In molar ratio.

presence of various concentrations of $\mathrm{SnCl}_{4}$ are shown in Figure 5. It was observed that the content of VAc in the copolymer increases with increasing the concentration of $\mathrm{SnCl}_{4}$ in the monomer feed. These data were analyzed to determine the apparent copolymerization parameters of VAc and AN using the FinemanRoss method $^{5}$ and Kelen-Tüdos method. ${ }^{6}$ The results of the apparent monomer reactivity ratios $r_{1}$ for VAc and $r_{2}$ for AN are represented in Table I. As shown the apparent copolymerization parameters are similar in the two methods. From Table I the value of $r_{1}$ for VAc increases on raising the concentration of $\mathrm{SnCl}_{4}$ in the reaction medium. It increases 
from 0.044 to 0.370 on increasing the concentration of $\mathrm{SnCl}_{4}$ from 0.0 to 0.153 . However, the value of $r_{2}$ for $\mathrm{AN}$ decreases with the addition of $\mathrm{SnCl}_{4}$ in the copolymerizing system. Moreover, the values of the apparent copolymerization parameters of the two monomers approach each other with increasing concentration of $\mathrm{SnCl}_{4}$ in the reaction medium $\left(r_{1}=r_{2}=0.37\right)$, indicating that $\mathrm{SnCl}_{4}$ increases the reactivity of VAc monomer towards the copolymerization process.

On the basis of the obtained results, the copolymerization of VAc and $\mathrm{AN}$ in the presence of $\mathrm{SnCl}_{4}$ can be considered a multicomponent system, since the two monomers can form complexes with $\mathrm{SnCl}_{4}$ and sixteen elementary polymerization reactions have to be taken into consideration during the propa- gation process.

\section{REFERENCES}

1. V. P. Zubov, L. I. Valuev, V. A. Kabanov, and V. A. Kargin, J. Polym. Sci., A-1, 9, 833 (1971).

2. M. Komiyama and H. Hirai, J. Polym. Sci., Polym. Chem. Ed., 14, 307, 627, 2009 (1976).

3. V. A. Kabanov, J. Polym. Sci., Polym. Symp., 67, 17 (1980).

4. B. M. Abo El-Khair, and S. M. Mokhtar, Commun, 30, 161 (1984).

5. M. Fineman and S. Ross, J. Polym. Sci., 5, 259 (1950).

6. T. Kelen and F. Tüdos, J. Makromol. Sci., Chem., A1, 1 (1975).

7. S. Takao, Ger. Offen. 2,933,680,09 Apr., 1981 [Chem. Abstr., 94, 2009912j (1981)].

8. S. Itoi, Maku, 6, 185 (1981) [Chem. Abstr., 95, 90415c (1981)]. 